\title{
Actin Polymerization Does Not Provide Direct Mechanical Forces for Vesicle Fission during Clathrin-Mediated Endocytosis
}

\author{
Li-Hua Yao, ${ }^{1}$ Yan Rao, ${ }^{1 \star}$ Chi Bang, ${ }^{2 \star}$ Svetlana Kurilova, ${ }^{3}$ Kelly Varga, ${ }^{1}$ Chun-Yang Wang, ${ }^{1}$ Brandon D. Weller, ${ }^{1}$ \\ Wonhwa Cho, ${ }^{3}$ Jun Cheng, ${ }^{2}$ and Liang-Wei Gong ${ }^{1}$ \\ Departments of ${ }^{1}$ Biological Sciences, ${ }^{2 B i o e n g i n e e r i n g, ~ a n d ~}{ }^{3}$ Chemistry, University of Illinois at Chicago, Chicago, Illinois 60607
}

Actin polymerization is important for vesicle fission during clathrin-mediated endocytosis (CME), and it has been proposed that actin polymerization may promote vesicle fission during CME by providing direct mechanical forces. However, there is no direct evidence in support of this hypothesis. In the present study, the role of actin polymerization in vesicle fission was tested by analyzing the kinetics of the endocytic tubular membrane neck (the fission-pore) with cell-attached capacitance measurements to detect CME of single vesicles in a millisecond time resolution in mouse chromaffin cells. Inhibition in dynamin GTPase activity increased the fission-pore conductance (Gp), supporting the mechanical role of dynamin GTPase in vesicle fission. However, disruptions in actin polymerization did not alter the fission-pore conductance $\mathrm{Gp}$, thus arguing against the force-generating role of actin polymerization in vesicle fission during CME. Similar to disruptions of actin polymerization, cholesterol depletion results in an increase in the fission-pore duration, indicating a role for cholesterol-dependent membrane reorganization in vesicle fission. Further experiments suggested that actin polymerization and cholesterol might function in vesicle fission during CME in the same pathway. Our results thus support a model in which actin polymerization promotes vesicle fission during CME by inducing cholesterol-dependent membrane reorganization.

\section{Introduction}

Clathrin-mediated endocytosis (CME) is the best-understood endocytic pathway that is critical for synaptic vesicle recycling, at least during mild stimulation (Granseth et al., 2006). Recruitments of clathrin-based coats at the plasma membrane induce CME, which consists of multiple steps: (1) invagination of the coated membrane to form a deeply invaginated bud, (2) constriction and fission of the endocytic tubular neck to separate the vesicle from the plasma membrane, and (3) inward movement of the vesicle into the cytosol (Kaksonen et al., 2006; Galletta et al., 2010). All of these energetically unfavorable steps require forcegenerating machinery to occur, and one of the proposed sources for the mechanical forces has been identified as actin polymerization. The force generated by actin polymerization is suggested to be necessary for endocytic invagination (Ferguson et al., 2009; Liu et al., 2009) by counteracting membrane tension (Aghamo-

Received May 22, 2013; revised Aug. 23, 2013; accepted Aug. 27, 2013.

Author contributions: L.-H.Y., Y.R., and L.-W.G. designed research; L.-H.Y., Y.R., C.B., S.K., K.V., and L.-W.G. performed research; L.-H.Y., Y.R., C.B., S.K., K.V., C.-Y.W., B.D.W., W.C., J.C., and L.-W.G. analyzed data; L.-W.G. wrote the paper.

This work was supported by Brain Research Foundation Award SF 2010-06 and National Science Foundation Award 1145581 to L.-W.G. and in part by Chicago Biomedical Consortium with support from the Searle Funds at the Chicago Community Trust to J.C.

The authors declare no competing financial interests.

*Y.R. and C.B. contributed equally to this work.

Correspondence should be addressed to Dr. Liang-Wei Gong, Department of Biological Sciences, University of Illinois at Chicago, 840 W. Taylor Street, Chicago, IL 60607. E-mail: Iwgong@uic.edu.

DOI:10.1523/JNEUROSCI.2171-13.2013

Copyright $\odot 2013$ the authors $\quad 0270-6474 / 13 / 3315793-06 \$ 15.00 / 0$ hammadzadeh and Ayscough, 2009) and for the departure of the internalized vesicle into the cytoplasm (Merrifield et al., 1999).

Actin polymerization may play key roles in vesicle fission during CME because actin polymerization burst reaches peak levels around the time of fission (Ehrlich et al., 2004; Merrifield et al., 2005). Furthermore, disruption of actin polymerization causes an increase in the number of endocytic vesicles that are unable to fully separate from the plasma membrane (Merrifield et al., 2005; Yarar et al., 2005). It has been therefore hypothesized that actin polymerization may promote vesicle fission by providing mechanical forces to constrict and/or elongate the endocytic tubular neck, and this hypothesis has been reiterated by a recent platinum replica electron microscopy study (Collins et al., 2011). However, the evidence in support of the force-generating role of actin polymerization in vesicle fission during endocytosis is lacking because it is technically challenging to directly measure the physical parameters associated with vesicle fission during endocytosis in living cells.

Our recent study has established a method to detect CME of single vesicles in mouse chromaffin cells using the cell-attached capacitance measurement (Yao et al., 2012). In these cellattached recordings with a sinusoidal voltage superimposed to the holding potential, a two-phase lock amplifier was used to analyze the changes of membrane capacitance in one channel and the changes of the membrane conductance in the other channel (Debus and Lindau, 2000). Before vesicle pinch-off when the tubular membrane neck of the endocytic vesicle has a low conductance, the vesicle capacitance $(\mathrm{Cv})$ is not fully charged, and there is a reduction of the value in the capacitance trace associated 
with a transient increase in the conductance. These changes in membrane capacitance and conductance can be used to estimate the kinetics of endocytic tubular neck, referred to as the endocytic fissionpore (Wu and $\mathrm{Wu}, 2007$; Yao et al., 2012). In the present study, we investigated the role of actin polymerization in vesicle fission during CME by using the cellattached capacitance measurement. Surprisingly, our result does not support the widely proposed force-generating function of actin polymerization during CME fission, prompting a reassessment of actin polymerization in vesicle fission during CME.

\section{Materials and Methods}

Chromaffin cell culture and cell-attached capacitance recordings. Adrenal glands of newborn mice of either sex were isolated in accordance with National Institutes of Health guidelines, as approved by the Animal Care and Use Committee of the University of Illinois at Chicago. Mouse chromaffin cells were prepared and cultured as previously described (Yao et al., 2012). Briefly, after incubation in papain solution at $37^{\circ} \mathrm{C}$ for $40 \mathrm{~min}$, adrenal glands were triturated gently through a $200 \mu$ l pipette tip. Cells plated on coverslips were incubated at $37^{\circ} \mathrm{C}$ in $5 \%$ $\mathrm{CO}_{2}$ and used within $4 \mathrm{~d}$.

For electrophysiological recordings, cells were bathed in solution with $140 \mathrm{~mm} \mathrm{NaCl}, 5$ $\mathrm{mm} \mathrm{KCl}, 2 \mathrm{~mm} \mathrm{CaCl}_{2}, 1 \mathrm{~mm} \mathrm{MgCl}_{2}, 10 \mathrm{~mm}$ HEPES, and $10 \mathrm{~mm}$ glucose. The cell-attached pipette solution contained $50 \mathrm{~mm} \mathrm{NaCl}, 100$ mM TEACl, $5 \mathrm{~mm} \mathrm{KCl}, 2 \mathrm{~mm} \mathrm{CaCl}_{2}, 1 \mathrm{~mm} \mathrm{MgCl}_{2}$, and $10 \mathrm{~mm}$ HEPES. The $\mathrm{pH}$ of the solutions was adjusted to 7.3 with $\mathrm{NaOH}$. Patch pipettes, coated with a sticky wax (Kerr), were fire-polished and had a typical resistance of $\sim 2 \mathrm{M} \Omega$. Cell-attached recordings were performed using an EPC-7 plus patch-clamp amplifier (HEKA-Elektronik). Changes of membrane capacitance and conductance were measured as described previously (Yao et al., 2012), with an SR830 lock-in amplifier (Stanford Research Systems) using a sine wave amplitude of $50 \mathrm{mV}$ (root mean square) at a frequency of $20 \mathrm{kHz}$. The output filter of the lock-in amplifier was set to a $1 \mathrm{~ms}$ time constant, $24 \mathrm{db}$.

The number of endocytic events per patch was counted as the total number of downward capacitance steps with sizes $>0.2 \mathrm{fF}$ within the first 5 min of recordings.

Fission-pore analysis. Endocytic fission-pore closures were analyzed as described previously (Yao et al., 2012). During fission-pore closure in which a transient increase in the Re signal was associated with a decline in the Im step, the vesicle capacitance (Cv) was determined as the total change in the Im trace for a particular event and the fission-pore conductance $(\mathrm{Gp})$ was calculated using the formula as follows: $\mathrm{Gp}=\frac{\omega \cdot C v}{\sqrt{\left(\frac{\omega \cdot C v}{\mathrm{Im}}\right)-1}}($ Yao et al., 2012). The fission-pore duration was defined as the time interval from the first point where $\mathrm{Gp}$ decreased to $<2 \mathrm{nS}$ and the final drop in Gp to zero. This final drop reflects the step response of the low pass filter setting of the lock-in amplifier ( $1 \mathrm{~ms}, 24 \mathrm{~dB}$ ). At this setting, $90 \%$ of the final value is reached within $\sim 7 \mathrm{~ms}$, so the last point of the fission-pore was taken as the time at 7-10 ms before the final drop to zero in the Gp trace. The fission-pore conductance $\mathrm{Gp}$ was taken as the average Gp value during the fission-pore duration time interval. Analysis of fissionpore kinetics were restricted to fission-pores with durations $>15 \mathrm{~ms}$ because shorter events were distorted by the lock-in amplifier lowpass filter (set to $1 \mathrm{~ms}, 24 \mathrm{~dB}$ ).

Reagents. We used dynasore at 20 or $40 \mu \mathrm{M}$ to inhibit dynamin GTPase activity (Macia et al., 2006), $1 \mu \mathrm{M}$ latrunculin B (LatB), and $4 \mu \mathrm{M}$ cytochalasin $\mathrm{D}(\mathrm{CytoD})$ to disrupt actin polymerization, and $5 \mathrm{~mm}$ methyl- $\beta$ cyclodextrin $(\mathrm{M} \beta \mathrm{CD})$ to extract membrane cholesterol. All these reagents, except dynasore, were purchased from Tocris Bioscience. Chromaffin cells were incubated with these different reagents for $60 \mathrm{~min}$ at room temperature before the recordings.

Statistical analysis. All the experiments were performed at room temperature. Data are expressed as mean \pm SEM, and Newman of one-way ANOVA tests was used for statistical analysis. Because typically one or two analyzable endocytic events can be obtained from each individual cells, every endocytic event was treated as statistically independent and the number of events was used for statistical testing of the fission-pore kinetics.

\section{Results}

The mechanical role of dynamin in vesicle fission was tested by investigating the effects of dynasore, a small molecule that inhibits dynamin GTPase activity (Macia et al., 2006), on the fissionpore kinetics (Fig. 1A). Dynasore treatment inhibited the number of endocytic events in a concentration-dependent manner $(p<$ 0.01 ) (Fig. $1 B$ ). Because very few endocytic events could be obtained from cells treated with $40 \mu \mathrm{M}$ dynasore (Fig. $1 B$ ), the effects of dynasore on the fission-pore kinetics were analyzed using a lower concentration at $20 \mu \mathrm{M}$. The $\mathrm{Cv}$ of the endocytic vesicles in cells treated with $20 \mu \mathrm{M}$ dynasore was comparable with that in control cells $(p>0.05)$ (Fig. $1 C)$, indicating that dynamin GTPase may not be critical in controlling the size of the endocytic 
A
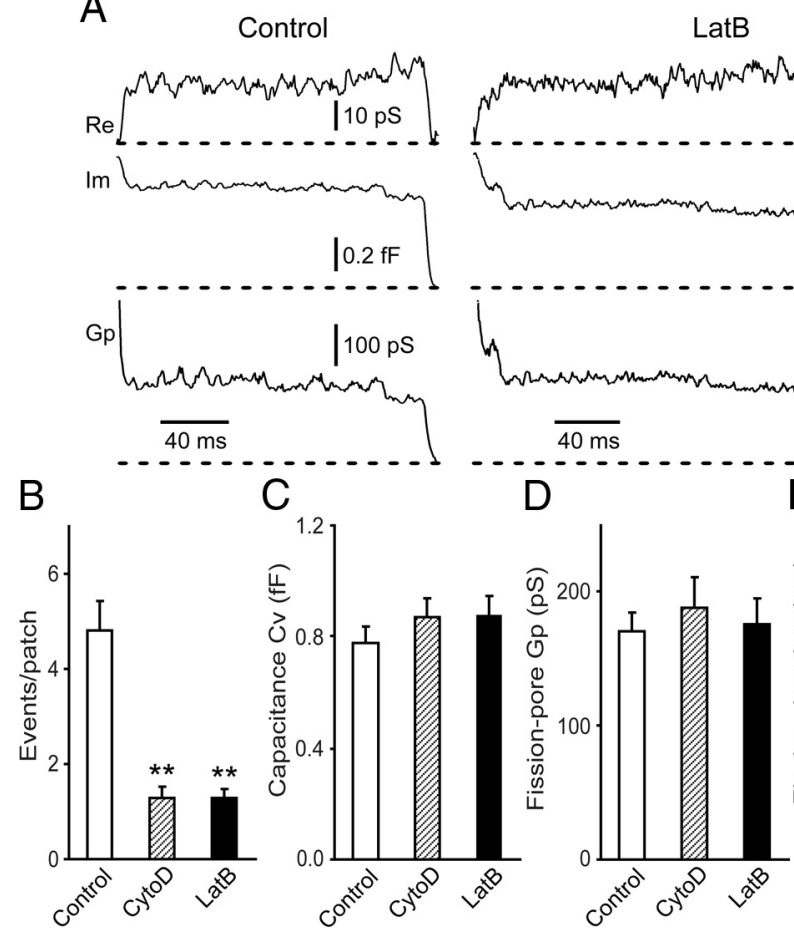

E

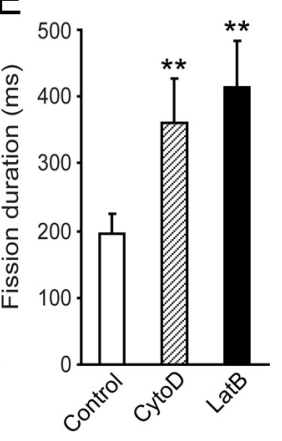

Figure 2. Effects on the fission-pore kinetics by LatB and CytoD to disrupt actin polymerization. $\boldsymbol{A}$, Representative fission-pore events from control cells (left) and cells treated with LatB (right). $\boldsymbol{B}$, The number of endocytic events was attenuated in cells treated with $4 \mu \mathrm{m}$ CytoD or $1 \mu \mathrm{m}$ LatB (Control: $n=58$ cells; LatB: $n=120$ cells; CytoD: $n=102$ cells; $p<0.01$ ). C $-\boldsymbol{E}$, C of endocytic vesicles $(\boldsymbol{C})$ and the fission-pore conductance $G p(D)$ were comparable between control cells ( $n=45$ events) and cells treated with $4 \mu \mathrm{m}$ CytoD ( $n=46$ events) or $1 \mu \mathrm{m}$ LatB $(n=44$ events) $(p>0.05)$, but the fission-pore duration was significantly increased in cells treated with CytoD or LatB $(p<0.01)(E) .{ }^{* *} p<0.01$.
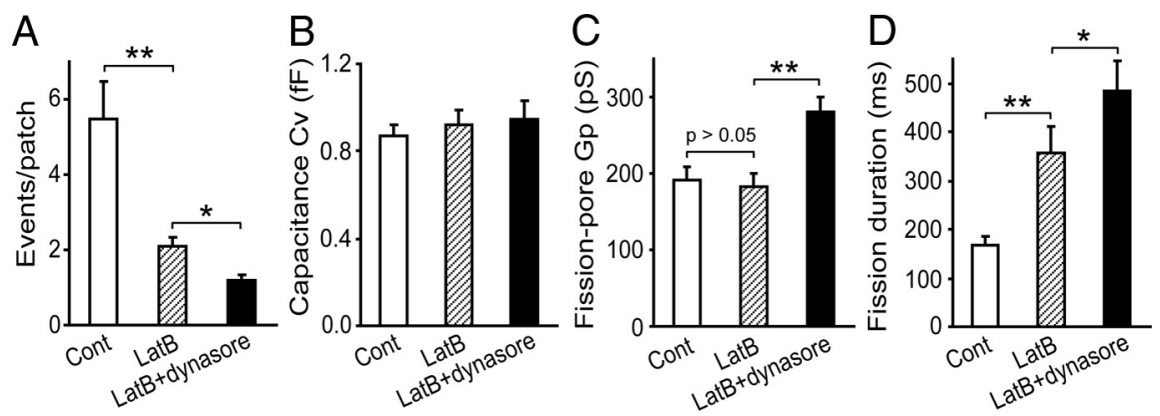

Figure 3. Additive effects of LatB and dynasore on the fission-pore kinetics. $A$, The number of endocytic events in cells preincubated with $1 \mu \mathrm{m}$ LatB was reduced by treatment with $20 \mu \mathrm{m}$ dynasore (Control: $n=48$ cells; LatB: $n=101$ cells; Lat $B+$ dynasore: $n=233$ cells; $p<0.05)$. $B$, Dynasore had no effects on the $(v$ of the endocytic vesicles in cells preincubated with LatB (Control: $n=58$ events; LatB: $n=52$ events; LatB + dynasore: $n=65$ events; $p>0.05) . C, D$, Dynasore treatment increased both the fission-pore conductance $\mathrm{Gp}(p<0.01)(\boldsymbol{C})$ and duration $(p<0.05)(\boldsymbol{D})$ in cells preincubated with LatB. ${ }^{*} p<0.05$. ${ }^{* *} p<0.01$.

vesicles. However, compared with control cells, cells treated with $20 \mu \mathrm{M}$ dynasore displayed a $60 \pm 14 \%$ increase in the fission-pore conductance $\mathrm{Gp}(p<0.01)$ (Fig. $1 D)$, suggesting a change in the geometry of the endocytic tubular membrane neck. In addition, compared with control cells, the fission-pore duration in dynasore-treated cells was increased by $42 \pm 21 \%(p<0.05)$ (Fig. $1 E$ ), suggesting that dynasore treatment slows down fission dynamics during CME.

Next, to witness the potential mechanical function of actin polymerization in endocytic fission, we examined the effects of LatB at $1 \mu \mathrm{M}$, which disrupts actin polymerization by sequestering actin monomers, on fission-pore kinetics (Fig. 2A). Com- pared with control cells, the number of endocytic events was substantially reduced in cells treated with LatB $(p<0.01)$ (Fig. $2 B$ ). The $\mathrm{Cv}$ of the endocytic vesicles was indistinguishable between control cells and cells treated with LatB $(p>0.05)$ (Fig. $2 C$ ), suggesting that actin polymerization may not be essential in determining the endocytic vesicle size. LatB treatment also had no obvious effect on the fission-pore conductance $\mathrm{Gp}(p>$ 0.05) (Fig. 2D), indicating no significant change in the geometry of the tubular membrane neck of the endocytic vesicle. However, LatB significantly increased the fission-pore duration $(p<0.01)$ (Fig. $2 E)$, suggesting that vesicle pinch-off is slower upon disruption in actin polymerization (Yarar et al., 2005). Meanwhile, treatment with CytoD at $4 \mu \mathrm{M}$, a drug that disrupts actin polymerization by binding to the barbed and fast-growing ends of F-actin, produced similar effects to LatB on the number of endocytic events (Fig. $2 B$ ), the Cv of the endocytic vesicle (Fig. $2 C)$, the fission-pore conductance $\mathrm{Gp}$ (Fig. 2D), and duration (Fig. 2E).

We next attempted to understand whether the effects of dynasore and LatB are additive by analyzing the effects of dynasore at $20 \mu \mathrm{M}$ on the fission-pore kinetics in the cells preincubated with LatB. Dynasore treatment further reduced the number of endocytic events in LatBpretreated cells with $38 \pm 5 \%$ endocytic events as control cell with no treatment, which is comparable with those in Figure $2 B(38 \pm 5 \% ; p<0.01)$ (Fig. $3 A)$. There was no change in the Cv of endocytic vesicles by dynasore in cells preincubated with LatB ( $p>0.05)$ (Fig. $3 B)$. However, dynasore treatment produced an increase in the fission-pore conductance $\mathrm{Gp}$ in LatB-preincubated cells $(p<0.05)$ (Fig. $3 C$ ). Moreover, $20 \mu \mathrm{M}$ dynasore also increased the fission-pore duration in cells pretreated with LatB $(p<0.05)$ (Fig. 3D). Collectively, these results suggest that the effects of dynasore and LatB on the fission-pore kinetics are additive.

Membrane cholesterol plays critical roles in clathrin-coated pit internalization (Rodal et al., 1999). A potential function of cholesterol in vesicle fission during CME was investigated by examining the fission-pore kinetics in cells treated with $\mathrm{M} \beta \mathrm{CD}$ (Fig. $4 A$ ) to acutely extract membrane cholesterol from the cell. As expected, $\mathrm{M} \beta \mathrm{CD}$ treatment induced a reduction in the number of endocytic events that can be detected $(p<0.01)$ (Fig. $4 B)$. The Cv of these endocytic vesicles in cells preincubated with $\mathrm{M} \beta \mathrm{CD}$ was comparable with that in control cells $(p>0.05)$ (Fig. $4 C)$, suggesting that cholesterol may not be essential in regulating the size of endocytic vesicles. Cells treated with $\mathrm{M} \beta \mathrm{CD}$ displayed no obvious changes in the fission-pore conductance Gp ( $p>0.05)$ (Fig. $4 D)$, indicating no obvious 
change in the fission-pore geometry. However, there was a substantial increase in the fission-pore duration in cells treated with $\mathrm{M} \beta \mathrm{CD}(p<0.01)$ (Fig. $4 E)$, suggesting that cholesterol may also be necessary for vesicle fission during CME.

Given the similar effects of LatB and $\mathrm{M} \beta \mathrm{CD}$ on the fission-pore kinetics (an increase in the fission-pore duration but no change in the fission-pore conductance $\mathrm{Gp}$ ), we reasoned that actin polymerization may regulate vesicle fission through cholesterol-dependent mechanisms, which was tested by examining the effects of LatB on the fission-pore kinetics in cells preincubated with $\mathrm{M} \beta \mathrm{CD}$. In $\mathrm{M} \beta \mathrm{CD}$ preincubated cells, LatB treatment caused no change in the number of endocytic events (Fig. $4 B$ ), the $\mathrm{Cv}$ of the endocytic vesicles (Fig. 4C), and the fission-pore conductance $\mathrm{Gp}(p>0.05)$ (Fig. $4 D)$. Moreover, in contrast to the LatB-induced increase in the fission-pore duration in control cells (Fig. 2E), LatB had no detectable effect in $\mathrm{M} \beta \mathrm{CD}$-preincubated cells $(p>0.05)$ (Fig. $4 E$ ), suggesting nonadditive effects of $M \beta C D$ and LatB on the fission-pore kinetics.

\section{Discussion}

Our data observe an increase in the fission-pore conductance Gp by dynasore. As a pore is widely assumed to be a cylindrical structure (Rosenboom and Lindau, 1994; $\mathrm{Wu}$ and $\mathrm{Wu}, 2007)$, the fission-pore conductance Gp can be calculated as follows: $\mathrm{Gp}=\frac{\pi}{4} \cdot \frac{\rho \cdot D_{\rho}^{2}}{L}$, where $\rho$, the bath solution conductivity, is a constant $(100 \Omega \times \mathrm{cm})$, $\mathrm{L}$ is the length of the fission-pore, and $\mathrm{D}_{\mathrm{p}}$ is the diameter of the fission-pore. It is obvious from this equation that the fission-pore conductance $\mathrm{Gp}$ is proportional to the square of the fission-pore diameter but inversely proportional to the fission-pore length. Therefore, the fission-pore with a higher $\mathrm{Gp}$ in cells treated with dynasore may represent a fission-pore with larger diameter, shorter length, or both, depending on whether dynamin functions as a pinchase by constriction/twisting (Roux et al., 2006), a poppase by elongating (Stowell et al., 1999), or both in vivo. The dynasore-induced increase in the fission-pore conductance Gp thus provides a direct piece of in vivo evidence supporting the force-generating role of dynamin GTPase in vesicle fission during CME. Recent studies have shown that, in addition to providing mechanical forces, dynamin may also drive vesicle fission by its dissociation from membrane tubes after dynamin disassembly is triggered by GTP hydrolysis (Pucadyil and Schmid, 2008). It is thus conceivable that the elongated fission-pore duration by dynasore we observed might be attributable to a slowdown of dynamin disassembly, although this phenotype could simply be a consequence of a weakening in the mechanical forces generated by dynamin GTPase.

A wider and/or shorter fission-pore upon dynamin inhibition, suggested by the increased fission-pore conductance by 20 $\mu \mathrm{M}$ dynasore we observed, seems to be conflicted with others' findings that expression of dynamin GTPase dominant-negative mutant (K44A) increases the tubulated clathrin-coated pit structures with narrow and long neck (Damke et al., 1994, 2001; Taylor et al., 2012). However, given the essential role of dynamin GTPase in vesicle fission during CME in mammalian cells, expression of dynamin GTPase dominant-negative mutant (K44A) will likely freeze endocytosis at endocytic stages prior to vesicle fission. Therefore, the tubulated clathrin-coated pits with narrow and long neck in cells expressing of dynamin GTPase dominantnegative mutant (K44A) likely represents a endocytic stage prior to vesicle fission of inefficient endocytic events (Damke et al., 1994, 2001; Taylor et al., 2012). Consistently, we found that dynasore at a higher concentration of $40 \mu \mathrm{M}$ blocks the number of endocytic events by $>90 \%$.

Our results point out an $\sim 60 \%$ decrease in the incidence of scission ( $\sim 2$ events/patch in LatB- or CytoD-treated cells and $\sim 5$ events/patch in control cells), which is comparable to an $\sim 50 \%$ decrease in the incidence of scission induced by LatB in fibroblasts as reported previously (Taylor et al., 2012). Additionally, our results demonstrate that actin polymerization disruption causes an increase in the fission-pore duration, reiterating the critical role of actin polymerization in vesicle fission during CME (Merrifield et al., 2005; Yarar et al., 2005). Mechanical force generated by actin cytoskeleton has been ambiguously hypothesized to constrict and/or elongate the tubular membrane neck of endocytic vesicles during CME. It has been recently found that, using platinum replica electron microscopy, the growing ends of the actin filaments are oriented toward the neck of the endocytic vesicles, proposing that actin polymerization regulates vesicle fission during CME mainly by providing mechanical forces to constrict the endocytic tubular neck (Collins et al., 2011). This proposal predicts an increase in the diameter of the endocytic tubular neck (the fission-pore) and thus an increase in the 
fission-pore conductance $\mathrm{Gp}$ upon actin polymerization disruption. However, our data demonstrate that, in contrast to the dynasore-induced increase in the fission-pore conductance $\mathrm{Gp}$, CytoD and LatB treatments did not alter the fission-pore conductance $\mathrm{Gp}$, thus arguing against the proposed force-generating role of actin polymerization in vesicle fission during CME.

Dynamin interacts with many actin regulatory proteins directly or indirectly (Orth and McNiven, 2003), suggesting a potential cross-link between functions of GTPase dynamin and actin polymerization in CME. A recent study has proposed a feedback mechanism between dynamin and actin polymerization in vesicle fission during CME: GTPase activity of dynamin regulates actin dynamics and, in turn, actin cytoskeleton is required to concentrate dynamin to the endocytic membrane neck (Taylor et al., 2012). However, our experiments indicate an independency in the functions of dynamin GTPase and actin polymerization in vesicle fission during CME based on two pieces of evidence: (1) differential effects of dynasore and LatB on the fission-pore kinetics (increases in both the fissionpore conductance $\mathrm{Gp}$ and duration by dynasore and an increase in the fission-pore duration with no change in the fission-pore conductance Gp by LatB); and (2) the effects of dynasore and LatB on the fission-pore kinetics are additive. Consistent with the independence in the functions of dynamin and actin polymerization in CME, there are robust recruitments of F-actin at clathrin-coated pits in the lack of dynamin (Ferguson et al., 2009) and a persistence of dynamindependent CME in the absence of actin (Yarar et al., 2005; Saffarian et al., 2009). The obligatory role of actin polymerization in fission of dynamin-independent CME in budding yeast (Kaksonen et al., 2006; Galletta et al., 2010) implies that the functions of GTPase dynamin and actin polymerization in CME may not depend on each other; at least the function of actin polymerization does not require dynamin.

Cholesterol plays critical roles in the formation of lipid phases or domains in membranes with multiple lipid components by membrane reorganization (Baumgart et al., 2003; Roux et al., 2005), and the line tension existing at the domain interfaces could induce the rupture of a lipid tubule (Liu et al., 2006). Recent studies have shown that the cholesteroldependent membrane reorganization is critical for fission of reconstituted membrane tubules (Baumgart et al., 2003; Roux et al., 2005) and Shiga toxin-triggered internalization, a clathrin-independent form of endocytosis (Römer et al., 2010). The $\mathrm{M} \beta \mathrm{CD}$-induced increase in the fission-pore duration we observed indicates that cholesterol, presumably cholesterol-dependent membrane reorganization, may also play critical roles in vesicle fission during CME.

Our results showed that $\mathrm{M} \beta \mathrm{CD}$ and $\mathrm{LatB}$ have similar effects on the fission-pore kinetics and that their effects on the fission-pore kinetics are nonadditive, implying cholesterol dependence in actin's role in vesicle fission during CME. It is thus interesting to speculate that actin polymerization may regulate vesicle during CME by inducing cholesterol-dependent membrane reorganizations. Consistently, cholesterol-dependent membrane reorganization induced by actin polymerization is essential for vesicle fission during Shiga toxin-triggered internalization, a clathrin-independent form of endocytosis (Römer et al., 2010). Indeed, it is shown that dynamic membrane-bound actin polymerization network can control when and where membrane domains form and may actively contribute to membrane reorganization in model lipid bilayers (Liu and Fletcher, 2006). However, it is possible that actin polymerization and cholesterol may function independently to support membrane reorganization that is critical for vesicle fission. A potential linker between actin polymerization and membrane reorganization could be phosphatidyl-4,5-bisphosphate $\left[\mathrm{PI}(4,5) \mathrm{P}_{2}\right]$ because many actinnucleating factors, such as N-WASP, interact with $\mathrm{PI}(4,5) \mathrm{P}_{2}$ and $\mathrm{PI}(4,5) \mathrm{P}_{2}$ dephosphorylation may play a critical role in endocytic fission by producing a boundary between a $\mathrm{PI}(4,5) \mathrm{P}_{2}$-rich and a $\mathrm{PI}(4,5) \mathrm{P}_{2}$-poor environment that could generate a line tension to assist the action of dynamin in this process (Liu et al., 2009; ChangIleto et al., 2011).

In conclusion, our results propose that actin polymerization may facilitate vesicle fission during CME by inducing cholesteroldependent membrane reorganization rather than providing direct mechanical forces to constrict and/or elongate the tubular neck of the endocytic vesicle.

\section{References}

Aghamohammadzadeh S, Ayscough KR (2009) Differential requirements for actin during yeast and mammalian endocytosis. Nat Cell Biol 11: 1039-1042. CrossRef Medline

Baumgart T, Hess ST, Webb WW (2003) Imaging coexisting fluid domains in biomembrane models coupling curvature and line tension. Nature 425:821-824. CrossRef Medline

Chang-Ileto B, Frere SG, Chan RB, Voronov SV, Roux A, Di Paolo G (2011) Synaptojanin 1-mediated PI (4,5)P2 hydrolysis is modulated by membrane curvature and facilitates membrane fission. Dev Cell 20:206-218. CrossRef Medline

Collins A, Warrington A, Taylor KA, Svitkina T (2011) Structural organization of the actin cytoskeleton at sites of clathrin-mediated endocytosis. Curr Biol 21:1167-1175. CrossRef Medline

Damke H, Baba T, Warnock DE, Schmid SL (1994) Induction of mutant dynamin specifically blocks endocytic coated vesicle formation. J Cell Biol 127:915-934. CrossRef Medline

Damke H, Binns DD, Ueda H, Schmid SL, Baba T (2001) Dynamin GTPase domain mutants block endocytic vesicle formation at morphologically distinct stages. Mol Biol Cell 12:2578-2589. CrossRef Medline

Debus K, Lindau M (2000) Resolution of patch capacitance recordings and of fusion pore conductances in small vesicles. Biophys J 78:2983-2997. CrossRef Medline

Ehrlich M, Boll W, Van Oijen A, Hariharan R, Chandran K, Nibert ML, Kirchhausen T (2004) Endocytosis by random initiation and stabilization of clathrin-coated pits. Cell 118:591-605. CrossRef Medline

Ferguson SM, Raimondi A, Paradise S, Shen H, Mesaki K, Ferguson A, Destaing O, Ko G, Takasaki J, Cremona O, O’Toole E, De Camilli P (2009) Coordinated actions of actin and BAR proteins upstream of dynamin at endocytic clathrin-coated pits. Dev Cell 17:811-822. CrossRef Medline

Galletta BJ, Mooren OL, Cooper JA (2010) Actin dynamics and endocytosis in yeast and mammals. Curr Opin Biotechnol 21:604-610. CrossRef Medline

Granseth B, Odermatt B, Royle SJ, Lagnado L (2006) Clathrin-mediated endocytosis is the dominant mechanism of vesicle retrieval at hippocampal synapses. Neuron 51:773-786. CrossRef Medline

Kaksonen M, Toret CP, Drubin DG (2006) Harnessing actin dynamics for clathrin-mediated endocytosis. Nat Rev Mol Cell Biol 7:404-414. CrossRef Medline

Liu AP, Fletcher DA (2006) Actin polymerization serves as a membrane domain switch in model lipid bilayers. Biophys J 91:4064-4070. CrossRef Medline

Liu J, Kaksonen M, Drubin DG, Oster G (2006) Endocytic vesicle scission by lipid phase boundary forces. Proc Natl Acad Sci U S A 103:10277-10282. CrossRef Medline

Liu J, Sun Y, Drubin DG, Oster GF (2009) The mechanochemistry of endocytosis. PLoS Biol 7:e1000204. CrossRef Medline

Macia E, Ehrlich M, Massol R, Boucrot E, Brunner C, Kirchhausen T (2006) Dynasore, a cell-permeable inhibitor of dynamin. Dev Cell 10:839-850. CrossRef Medline

Merrifield CJ, Moss SE, Ballestrem C, Imhof BA, Giese G, Wunderlich I, Almers W (1999) Endocytic vesicles move at the tips of actin tails in cultured mast cells. Nat Cell Biol 1:72-74. CrossRef Medline

Merrifield CJ, Perrais D, Zenisek D (2005) Coupling between clathrincoated-pit invagination, cortactin recruitment, and membrane scission observed in live cells. Cell 121:593-606. CrossRef Medline 
Orth JD, McNiven MA (2003) Dynamin at the actin-membrane interface. Curr Opin Cell Biol 15:31-39. CrossRef Medline

Pucadyil TJ, Schmid SL (2008) Real-time visualization of dynamincatalyzed membrane fission and vesicle release. Cell 135:1263-1275. CrossRef Medline

Rodal SK, Skretting G, Garred O, Vilhardt F, van Deurs B, Sandvig K (1999) Extraction of cholesterol with methyl-beta-cyclodextrin perturbs formation of clathrin-coated endocytic vesicles. Mol Biol Cell 10:961-974. CrossRef Medline

Römer W, Pontani LL, Sorre B, Rentero C, Berland L, Chambon V, Lamaze C, Bassereau P, Sykes C, Gaus K, Johannes L (2010) Actin dynamics drive membrane reorganization and scission in clathrin-independent endocytosis. Cell 140:540-553. CrossRef Medline

Rosenboom H, Lindau M (1994) Exo-endocytosis and closing of the fission pore during endocytosis in single pituitary nerve terminals internally perfused with high calcium concentrations. Proc Natl Acad Sci U S A 91: 5267-5271. CrossRef Medline

Roux A, Cuvelier D, Nassoy P, Prost J, Bassereau P, Goud B (2005) Role of curvature and phase transition in lipid sorting and fission of membrane tubules. EMBO J 24:1537-1545. CrossRef Medline

Roux A, Uyhazi K, Frost A, De Camilli P (2006) GTP-dependent twisting of dynamin implicates constriction and tension in membrane fission. $\mathrm{Na}-$ ture 441:528-531. CrossRef Medline

Saffarian S, Cocucci E, Kirchhausen T (2009) Distinct dynamics of endocytic clathrin-coated pits and coated plaques. PLoS Biol 7:e1000191. CrossRef Medline

Stowell MH, Marks B, Wigge P, McMahon HT (1999) Nucleotidedependent conformational changes in dynamin: evidence for a mechanochemical molecular spring. Nat Cell Biol 1:27-32. CrossRef Medline

Taylor MJ, Lampe M, Merrifield CJ (2012) A feedback loop between dynamin and actin recruitment during clathrin-mediated endocytosis. PLoS Biol 10:e1001302. CrossRef Medline

Wu W, Wu LG (2007) Rapid bulk endocytosis and its kinetics of fission pore closure at a central synapse. Proc Natl Acad Sci U S A 104:10234-10239. CrossRef Medline

Yao LH, Rao Y, Varga K, Wang CY, Xiao P, Lindau M, Gong LW (2012) Synaptotagmin 1 is necessary for the $\mathrm{Ca}^{2+}$ dependence of clathrinmediated endocytosis. J Neurosci 32:3778-3785. CrossRef Medline

Yarar D, Waterman-Storer CM, Schmid SL (2005) A dynamic actin cytoskeleton functions at multiple stages of clathrin-mediated endocytosis. Mol Biol Cell 16:964-975. CrossRef Medline 